\title{
Radiation Properties of Spherical Antennas as a Function of the Location of the Driving Force
}

\author{
Philip R. Karr
}

\begin{abstract}
A theoretical analysis is made of the radiation properties of a perfectly conducting sphere excited as an antenna by a driving force that acts in the $\theta$ direction in a narrow strip around the circle of colatitude $\theta=\theta_{0}$ on the surface of the sphere. As the location $\theta_{0}$ is varied, the radiation pattern, together with the current distribution and the input admittance, change. In the present paper some of these effects are analyzed, it is believed for the first time. In previous work only the case of center feed is treated.

Unless $\theta_{0}$ equals 90 degrees, the radiation pattern is not symmetrical with respect to the equatorial plane. Some interesting features of this asymmetry are discovered in the course of the analysis that have also been found experimentally in certain classes of antennas driven asymmetrically. The radiation conductance, which is proportional to the amount of power radiated for a given applied voltage, varies in a complicated way with $\theta_{0}$. However, for a sphere whose radius is not too large compared with the wavelength, this trend is approximately as $\sin ^{4} \theta_{0}$. In some cases the radiation conductance is not a maximum for $\theta_{0}=90$ degrees, but is greater when the feeding strip is somewhat to one side of the equatorial plane. Other features of the radiation are analyzed. Reasons are given for believing that the idealization of the feed conditions does not prevent the theory from giving experimentally pertinent results for the radiation properties.
\end{abstract}

\section{Introduction}

The center-fed antenna in various shapes has been the subject of considerable theoretical and experimental research. On the other hand, the study of antennas not necessarily fed at the center has apparently received little attention in the theoretical literature. There has recently been a certain amount of systematic experimental work in this field $[1,2]^{2}$, and there are a number of practical applications of such antennas $[3,4]$. It therefore appears to be of some interest to attempt a theoretical study of asymmetrically fed antennas. In attempting such a study, it is necessary to find an idealization of the problem that can be solved mathematically and at the same time yields some physical insight into the nature of the results. In the present paper the spherical antenna is chosen for study. This form has the advantage that an idealized boundary value problem duplicating actual conditions to a certain extent can be set up and solved.

The spherical antenna, while not expected to be a popular type for applications, is in a number of respects not radically different from more conventional antennas, so that the results obtained can be of qualitative assistance in the study of such antennas. The problem of the prolate spheroid antenna with asymmetrical feed can in principle be solved as a boundary value problem in a manner similar to that presented here for the sphere, but tables of spheroidal wave functions are at present rather inadequate for any detailed study. The present report is in some respects an extension of the

1 A dissertation submitted to the Faculty of the Graduate School of Arts and Sciences of the Catholic University of America in partial fulfillment of the requirements for the degree of Doctor of Philosophy.

2 Figures in brackets indicate the literature references at the end of this paper. work of Stratton and Chu [5] on the spherical center-fed antenna. A number of the equations. found are generalizations of corresponding equations already found for the center-fed antenna. The generalization of the location of the feed introduces some interesting effects. These, together with some other matters, are discussed in some detail. It is hoped that this paper will help to stimulate some further work on the subject.

\section{Statement of the Problem and Its Formal Solution}

We consider a conducting sphere, figure 1 , of radius $a$, excited as an antenna by a driving emf, which acts in the $\theta$ direction in a narrow strip around the circle of colatitude $\theta=\theta_{0}$ on the surface of the sphere. The center of the sphere is the origin of a system of spherical coordinates, $r, \theta, \phi$. As the location $\theta_{0}$ is varied, the radiation pattern, together with the current distribution and the input admittance, change. The purpose of this paper is to describe and, as far as possible, to "understand" some of these effects. Stating the boundary conditions more precisely, we are applying a field $E^{\prime}$ across a small zone of the conducting spherical surface. This causes a field $E$ to appear in space. The value of the tangential component $E_{\theta}$ of $E$ on the surface is such that $E_{\theta}+E^{\prime}=0$ everywhere on the spherical surface since we shall assume perfect conductivity. The solution of the boundary value problem consists in finding wave solutions of Maxwell's equations, which on the surface of the antenna reduce to $E_{\theta}=-E^{\prime}$. 
We consider wave solutions of Maxwell's equations in the charge-free and current-free space around the sphere that will satisfy the correct boundary conditions on the sphere. The appropriate solutions (eq 4) will be in the form of rather well-known spherical fields. However, for the sake of completeness we shall derive these solutions. Let the medium be characterized by $\mu, \epsilon$, and let the time variation be as $e^{j_{\omega} t}$. Then Maxwell's equations in mks rationalized units reduce to

$$
\begin{gathered}
\nabla \times \boldsymbol{E}=-j \omega \mu \boldsymbol{H}, \\
\nabla \times \boldsymbol{H}=j \omega \epsilon \boldsymbol{E} .
\end{gathered}
$$

(The divergence equations are automatically satisfied in this case by solutions of (1) and (2), since the divergence of the curl of a vector is zero). In spherical coordinates, (1) and (2) become

$$
\left.\begin{array}{c}
\frac{1}{r \sin \theta}\left[\frac{\partial}{\partial \theta}\left(E_{\phi} \sin \theta\right)-\frac{\partial E_{\theta}}{\partial \phi}\right]=-j \omega \mu H_{r} \\
\frac{1}{r}\left[\frac{1}{\sin \theta} \frac{\partial E_{r}}{\partial \phi}-\frac{\partial}{\partial r}\left(r E_{\phi}\right)\right]=-j \omega \mu H_{\theta} \\
\frac{1}{r}\left[\frac{\partial}{\partial r}\left(r E_{\theta}\right)-\frac{\partial E_{r}}{\partial \theta}\right]=j \omega \mu H_{\phi} \\
\frac{1}{r \sin \theta}\left[\frac{\partial}{\partial \theta}\left(H_{\phi} \sin \theta-\frac{\partial H_{\theta}}{\partial \phi}\right]=j \omega \epsilon E_{r}\right. \\
\frac{1}{r}\left[\frac{1}{\sin \theta} \frac{\partial H_{r}}{\partial \phi}-\frac{\partial}{\partial r}\left(r H_{\phi}\right)\right]=j \omega \epsilon E_{\theta} \\
\frac{1}{r}\left[\frac{\partial}{\partial r}\left(r H_{\theta}\right)-\frac{\partial H_{r}}{\partial \theta}\right]=j \omega \epsilon E_{\phi}
\end{array}\right\}
$$

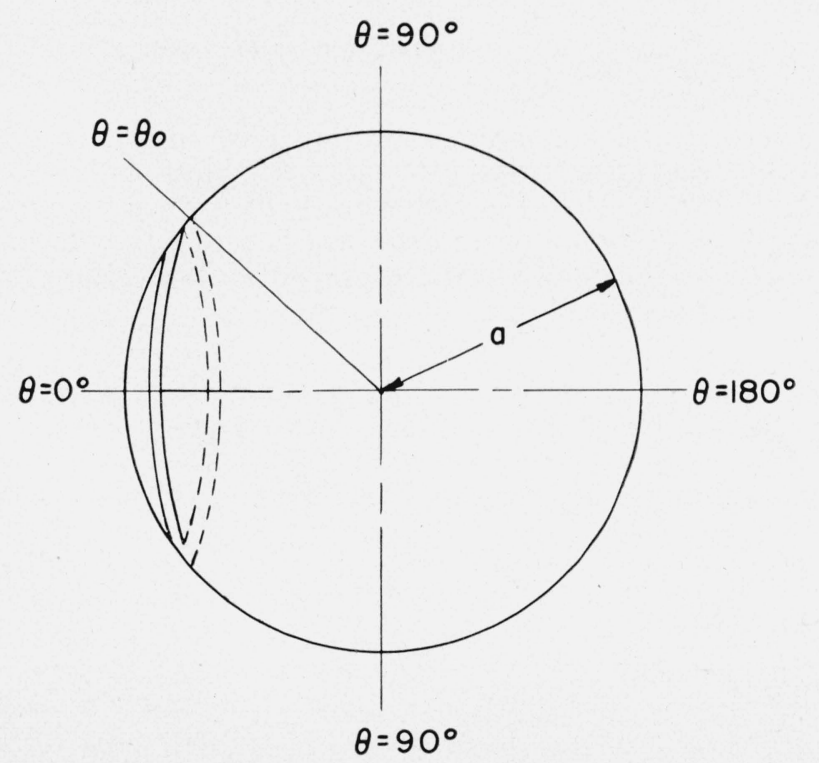

Figure 1. Spherical antenna.

The applied emf acts in the $\theta$ direction in a narrow zone around the sphere at $\theta=\theta_{0}$.
Now in the present problem it is clear that $\partial / \partial \phi=0$. Using this relation in (1a) and (2a), we find immediately that the equations split into two sets of equations, one containing only $H_{\phi}, E_{\theta}$, and $E_{r}$, the other containing only $E_{\phi}, H_{\theta}$, and $H_{r}$. Solutions of these two sets are known as TM (transverse magnetic) and TE (transverse electric) solutions, respectively. In the present problem, it is clear that the TE solutions are inappropriate. We shall be able to satisfy our conditions with $T M$ solutions; that is, solutions for which the magnetic lines are circles in planes perpendicular to the polar axis with their centers on this axis, and for which the electric lines lie in meridian planes.

Write the three equations containing $H_{\phi}, E_{\theta}, E_{r}$ :

$$
\begin{aligned}
& \frac{\partial}{\partial r}\left(r E_{\theta}\right)-\frac{\partial E_{r}}{\partial \theta}=-j \omega \mu\left(r H_{\phi}\right) . \\
& \frac{1}{r \sin \theta} \frac{\partial}{\partial \theta}\left(H_{\phi} \sin \theta\right)=j \omega \epsilon E_{r}, \\
& -\frac{\partial}{\partial r}\left(r H_{\phi}\right)=j \omega \epsilon\left(r E_{\theta}\right) .
\end{aligned}
$$

When we differentiate the last two equations, with respect to $\theta$ and $r$, respectively, and substitute in the first, we obtain

$\frac{\partial^{2}}{\partial r^{2}}\left(r H_{\phi}\right)+\frac{1}{r^{2}} \frac{\partial}{\partial \theta}\left[\frac{1}{\sin \theta} \frac{\partial}{\partial \theta}\left(r H_{\phi} \sin \theta\right)\right]+k^{2}\left(r H_{\phi}\right)=0$,

where

$$
k=\omega \sqrt{\mu \epsilon}=\frac{2 \pi}{\lambda} .
$$

This equation is separable in the form $r H_{\phi}=R(r) \Theta(\theta)$. Calling the separation constant $n(n+1)$, we obtain

$$
\frac{r^{2}}{R} \frac{\partial^{2} R}{\partial r^{2}}+k^{2} r^{2}=-\frac{1}{\Theta} \frac{d}{d \theta}\left[\frac{1}{\sin \theta} \frac{d}{d \theta}(\Theta \sin \theta)\right]=n(n+1) .
$$

Making the substitution $x=\cos \theta$, the equation for $\theta$ becomes

$$
\left(1-x^{2}\right) \frac{d^{2} \Theta}{d x^{2}}-2 x \frac{\partial \Theta}{\partial x}+\left[n(n+1)-\frac{1}{1-x^{2}}\right] \Theta=0,
$$

which is the equation for the associated Legendre functions satisfied by the functions $P_{n}^{1}(x)$ and $Q_{n}^{1}(x)$ [6]. Only $P_{n}^{1}(x)$ can be used in our problem, because the function $Q_{n}^{1}(x)$ has singularities at $x= \pm 1$.

Returning to the $r$ equation, we find that the substitution $P=R / \sqrt{r}$ yields

$$
\frac{\partial^{2} P}{\partial r^{2}}+\frac{1}{r} \frac{\partial P}{\partial r}+\left[k^{2}-\frac{\left(n+\frac{1}{2}\right)^{2}}{r^{2}}\right] P=0
$$

which is Bessel's differential equation of order $n+\frac{1}{2}$, 
whose general solution is of the form

$$
P=C_{n} J_{n+\frac{1}{2}}(k r)+D_{n} N_{n+\frac{1}{2}}(k r) .
$$

Since we desire the solution to approach a travelling wave as $r \rightarrow \infty$, we use the linear combination (Hankel function),

$$
H_{n+\frac{1}{2}}^{(2)}(k r)=J_{n+\frac{1}{2}}(k r)-j N_{n+\frac{1}{2}}(k r) .
$$

We then have as our generic $T M$ solution, after some manipulation,

$$
\begin{gathered}
H_{\phi}=A_{n} r^{-1 / 2} P_{n}^{1}(\cos \theta) H_{n+\frac{1}{2}}^{(2)}(k r), \\
E_{\theta}=\frac{A_{n}}{j \omega \epsilon} r^{-3 / 2} P_{n}^{1}(\cos \theta)\left[n H_{n+\frac{1}{2}}^{(2)}(k r)-k r H_{n-\frac{1}{2}}^{(2)}(k r)\right], \\
E_{r}=\frac{A_{n}}{j \omega \epsilon} \frac{r^{-3 / 2}}{\sin \theta} \frac{\partial}{\partial \theta} P_{n}^{1}(\cos \theta)\left[H_{n+\frac{1}{2}}^{(2)}(k r)\right] .
\end{gathered}
$$

These are the fields of electric dipoles, quadrupoles, and in general $2^{n}$-poles. The $T E$ solutions, not used here, represent the fields of magnetic $2^{n}$-poles.

Our solution will be a superposition of $T M$ waves (eq 4). To find the coefficients $A_{n}$ we expand the applied field $E^{\prime}$ in a series of associated Legendre polynomials $P_{n}^{1}(\cos \theta)$ :

$$
E^{\prime}(\theta)=\frac{V}{a} \sum_{n=1}^{\infty} a_{n} P_{n}^{1}(\cos \theta) .
$$

In (5) we have introduced the applied voltage $V$ defined by

$$
V=\int_{0}^{\pi} E^{\prime}(\theta) a d \theta
$$

The letter $a$, it will be recalled, stands for the radius of the sphere. This normalization allows the $a_{n}$ to be pure numbers, whereas $E^{\prime}(\theta)$ will have the dimensions of field strength (volts per meter). Multiplying (5) by $P_{n}^{1}(\cos \theta) \sin \theta d \theta$, and integrating from 0 to $\pi$, we have, on account of the orthogonality and normalization properties of $P_{n}^{1}(\cos \theta)$, the following expression for $a_{n}$ :

$$
\frac{V}{a} a_{n}=\frac{2 n+1}{2 n(n+1)} \int_{0}^{\pi} E^{\prime}(\theta) P_{n}^{1}(\cos \theta) \sin \theta d \theta
$$

Now from (4),

$\left.\left.E_{\theta}\right|_{r=a}=\frac{a^{-3 / 2}}{j \omega \epsilon} \sum_{n=1}^{\infty} A_{n} P_{n}^{1} \cos \theta\right)\left[n H_{n+\frac{1}{2}}^{(2)}(k a)-k a H_{n+\frac{1}{2}}^{(2)}(k a)\right]$

Comparing (8) and (5) and remembering that on the surface, $E_{\theta}=-E^{\prime}$, we have for $A_{n}$ :

$$
\frac{a}{V} A_{n}=-j \omega \epsilon a^{3 / 2} a_{n}\left[n H_{n+\frac{1}{2}}^{(2)}(k a)-k a H_{n+\frac{1}{2}}^{(2)}(k a)\right]^{-1}
$$

Thus the solution of our problem is a superposition of the waves given in eq 4 , with the $A_{n}$ given by eq 9 . The manner of deriving the $A_{n}$ guarantees that the boundary conditions are satisfied.

The values of the $a_{n}$ and therefore of the $A_{n}$, depend upon the assumed form of $E^{\prime}(\theta)$. In most of what follows we shall specialize by assuming $E^{\prime}(\theta)$ takes the form of a "delta" function applied at the feed point $\theta=\theta_{0}$ :

$$
E^{\prime}(\theta)=\frac{V}{a} \delta\left(\theta-\theta_{9}\right)
$$

This means that

$$
a_{n}=\frac{2 n+1}{2 n(n+1)} P_{n}^{1}\left(\cos \theta_{0}\right) \sin \theta_{0} .
$$

It should be noted that if $E^{\prime}(\theta)$ is applied over a small finite zone and does not vary wildly with $\theta$ over the zone, then the $a_{n}$ found in such a case would be practically the same as in eq 11 except for high values of $n$ (cf. eq 53 to 55). For this reason, results based upon (11), which are correct for an infinitesimal zone are almost correct for a small finite zone, except for those results that depend upon high values of $n$.

\section{The Radiation Field}

\section{General Relations}

Having written down the formal solution of our problem, we shall now investigate in more detail some of the important characteristics of our antenna. Let us first consider the "radiation field", (that part of the field that persists at large values of $r$ ). We note that as $r \rightarrow \infty$, the second term of (4b) dominates, and

$$
E_{r a d}=\sum_{n=1}^{\infty} \frac{A_{n}}{j \omega \epsilon} r^{-1 / 2} k P_{n}^{1}(\cos \theta) H_{n-\frac{1}{2}}^{(2)}(k r),
$$

where $E_{r a d}$ denotes the radiation part of $E_{\theta}$.

We see also, from (4b) and 4c) that as $r \rightarrow \infty$, $E_{r} / E_{\theta} \rightarrow 0$, so that the radiation field is purely transverse (as it should be). Now as $r$ becomes very large, we have the well-known asymptotic expression for the Hankel function:

$$
H_{n-\frac{1}{2}}^{(2)}(k r) \rightarrow \sqrt{\frac{2}{\pi k r}} e^{-j k r} e^{(j n \pi) / 2}=j^{n} \sqrt{\frac{2}{\pi k r}} e^{-j k r} .
$$

Combining (13), (12), and (9), we find

$$
\frac{E_{r a d}}{V}=\sqrt{\frac{2 k a}{\pi}} \frac{e^{-j k r}}{r} \sum_{n=1}^{\infty} a\left(n, \theta_{0}\right) L(n, k a) P_{n}^{1}(\cos \theta),
$$

where

$$
\begin{aligned}
a\left(n, \theta_{0}\right) & \equiv a_{n} \\
& =\frac{2 n+1}{2 n(n+1)} P_{n}^{1}\left(\cos \theta_{0}\right) \sin \theta_{0},(\text { using }(11)),
\end{aligned}
$$


and

$$
L(n, k a) \equiv j^{n}\left[k a H_{n-\frac{1}{2}}^{(2)}(k a)-n H_{n-\frac{1}{2}}^{(2)}(k a)\right]^{-1} .
$$

We find also, in the radiation region

$$
H_{\phi}=\sqrt{\frac{\epsilon}{\mu}} E_{\theta} \equiv \frac{1}{Z_{0}} E_{\theta} .
$$

Thus the radiation field decreases as $1 / r$, as it should. Its $\theta$ variation is a superposition of the functions $P_{n}^{1} \quad(\cos \theta)$. The contribution of each order $n$ depends upon the nature and location of the excitation through $a\left(n, \theta_{0}\right)$ and upon the size of the sphere through $L(n, k a)$.

Before considering typical radiation patterns, it is useful to investigate the convergence of the series for $E_{\text {rad }}$. We have for $n \gg x$, the Debye asymptotic formula [7]

$H_{n}^{(2)}(x) \sim \sqrt{\frac{2}{\pi x \sinh \alpha}} \exp \left[x(\alpha \cosh \alpha-\sinh \alpha)+j \frac{\pi}{2}\right]$,

where $n=x \cosh \alpha$.

Writing $\mathrm{x} / \mathrm{n}=\operatorname{sech} \alpha=\zeta$, we obtain

$$
\begin{aligned}
H_{n}^{(2)}(x)= & \sqrt{\frac{2}{\pi n\left(1-\frac{1}{\zeta^{2}}\right)^{1 / 2}}} \exp \left\{j \frac{\pi}{2}+n \sqrt{1-\zeta^{2}}\right. \\
& {\left.\left[\frac{1}{\zeta} \ln \left(\frac{1}{\zeta}+\sqrt{\frac{1}{\zeta^{2}}-1}\right)-\sqrt{\frac{1}{\zeta^{2}}-1}\right]\right\} }
\end{aligned}
$$

Disregarding small terms, the expression in braces becomes

$$
\sim j \frac{\pi}{2}+n\left(\frac{1}{\zeta} \ln \frac{2}{\zeta}-\frac{1}{\zeta}\right)
$$

so we find the approximate expression

$$
H_{n}^{(2)}(x) \sim j \sqrt{\frac{2}{\pi n}}\left(\frac{2 n}{x e}\right)^{n^{2} / x} \quad x \ll n .
$$

Substituting in (16) and disregarding small terms, we find

$$
L(n, k a) \approx-j^{n-1} \sqrt{\frac{n}{2 n}}\left(\frac{2 n+1}{k a e}\right)^{-\frac{\left(n+\frac{1}{2}\right)^{2}}{k a}} .
$$

For the associated Legendre polynomial we have the asymptotic formula [8], for $n$ large

$$
P_{n}^{1}(\cos \theta) \approx \frac{2 n^{1 / 2}}{\sqrt{2 \pi \sin \theta}} \cos \left[\left(n+\frac{1}{2}\right) \theta+\frac{\pi}{4}\right] .
$$

One sees then that on account of the factor

$$
\left(\frac{2 n+1}{k a e}\right)^{-\frac{\left(n+\frac{1}{2}\right)^{2}}{k a}}=\left(\frac{2 n+1}{k a e}\right)^{-\frac{(2 n+1)^{2}}{4 k a}}
$$

in (19), the series (14) for $E_{\text {rad }}$ is convergent and that the terms fall off exceedingly rapidly as $n$ becomes large. In physical terms, this means that the highorder modes contribute negligibly to the radiation. In practice we find that we may terminate the series around $n \approx 2 k a$. This can be seen roughly by examining figures 2 ,a and $2, \mathrm{~b}$, which show the real and imaginary parts of $L(n, k a)$ plotted versus $k a$.
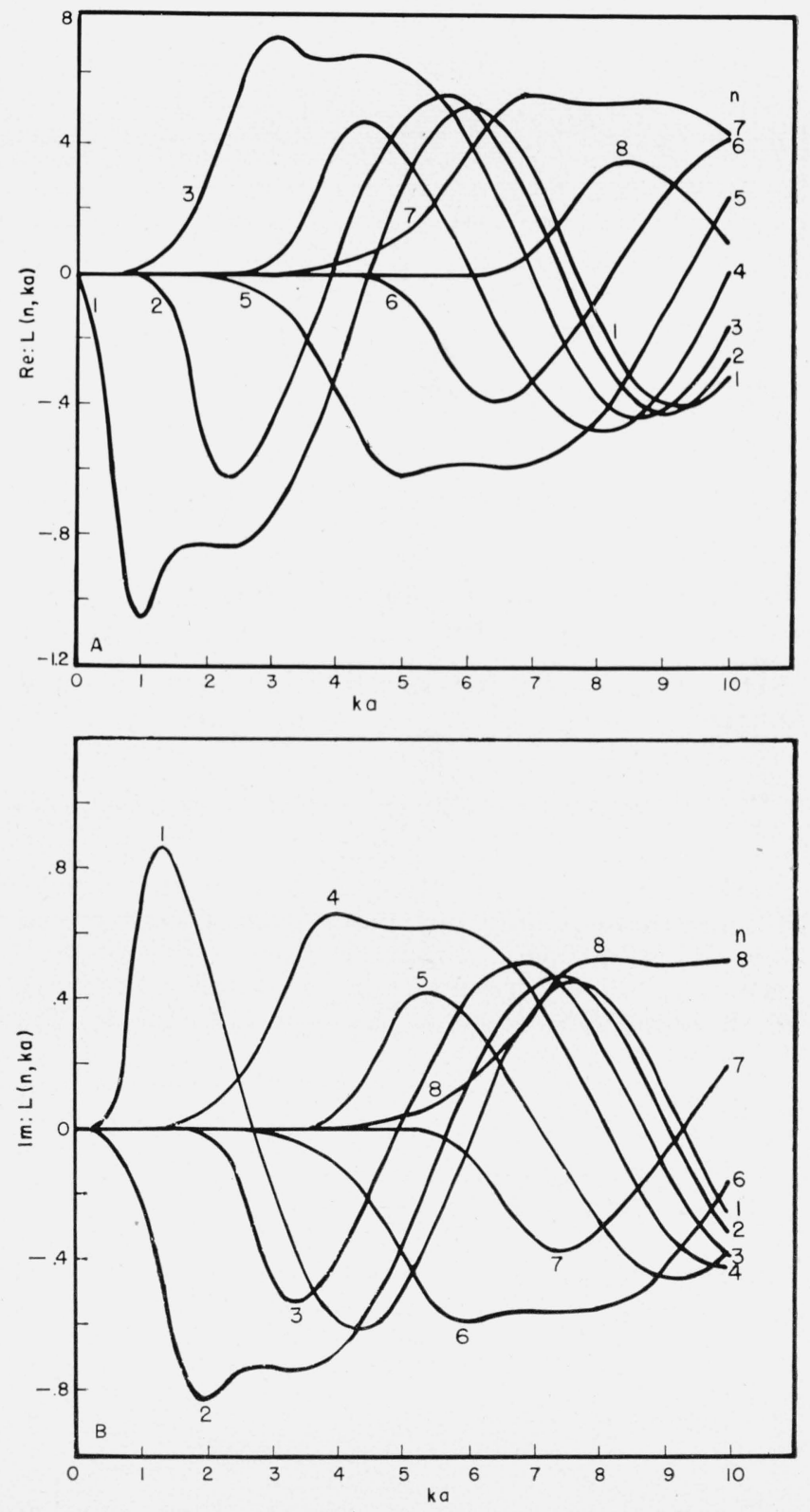

Figure 2. $L(n, k a)$ as a function of ka for several values of $n$. A, real part; B, imaginary part. 


\section{Small Spheres}

It is of some interest to study the radiation from a small sphere $(k a \ll 1)$. We expect on general grounds that as the radiating system becomes smaller (relative to the wavelength), the lowest (dipole) mode should become dominant. This is confirmed by an examination of figures 2 ,a and $2, b$; as $k a$ decreases considerably below 1 , only 1 mode (represented by the real part of $L(1, k a)$ ) contributes appreciably.

We may find an explicit expression for the effective dipole moment of the small spherical antenna. We have

$$
\begin{aligned}
& H_{1 / 2}^{(2)}(k a)=j \sqrt{\frac{2}{\pi k a}} e^{-j k a} \\
& H_{3 / 2}^{(2)}(k a)=\sqrt{\frac{2}{\pi k a}} e^{-j k a}\left(\frac{j}{k a}-1\right)
\end{aligned}
$$

as $k a \rightarrow 0$, these become

$$
\begin{aligned}
& H_{1 / 2}^{(2)}(k a) \approx j \sqrt{\frac{2}{\pi k a}}, \\
& H_{3 / 2}^{(2)}(k a) \approx \frac{j}{k a} \sqrt{\frac{2}{\pi k a}},
\end{aligned}
$$

so that $L(1, k a) \equiv j\left[k a j \sqrt{\frac{2}{\pi k a}}-\frac{j}{k a} \sqrt{\frac{2}{\pi k a}}\right]^{-1}$

$$
\approx-\sqrt{\frac{\pi}{2}}(k a)^{3 / 2} \quad k a \ll 1 .
$$

One then finds, for the limiting case of small spheres,

$$
\frac{E_{r a d}}{V} \approx-(k a)^{2} \frac{e^{-j k r}}{r} \frac{3}{4} P_{1}^{1}(\cos \theta) \sin \theta_{0} P_{1}^{1}\left(\cos \theta_{0}\right),
$$

a radiation pattern like that for the Hertzian dipole. fat Since there is only one mode, the off-center feed does not introduce asymmetry in the radiation pattern, but merely reduces the field radiated for a given applied voltage in the ratio $P_{1}^{1}\left(\cos \theta_{0}\right) \sin$ $\theta_{0}: 1=\sin ^{2} \theta_{0}: 1$. $M$ is

The radiation field of a dipole of dipole moment

$$
E_{\text {rad }}=\frac{-M k^{2}}{4 \pi \epsilon} \frac{e^{-j k r}}{r} \sin \theta \quad[9]
$$

Comparing this with (22) we find that the effective dipole moment of the small sphere is

$$
M=3 \pi \epsilon V a^{2} \sin ^{2} \theta_{0} .
$$

\section{Moderate-Sized Spheres}

We now proceed to investigate the radiation patterns of spheres comparable in size to a waveength. In figures 3,4 , and 5, we have plotted the power radiation patterns $\left|E_{r a d}\right|^{2}$, all normalized to the same maximum value of $\left|E_{r a d}\right|^{2}$, for $k a=1,1 \frac{1}{2}$, and 2 , and several values of $\theta_{0}$. An examination of these patterns discloses an interesting feature, which may be described roughly as follows: as the feedpoint $\theta_{0}$ is moved towards $0^{\circ}$, the patterns become more asymmetrical and tend to lean more and more away from the feedpoint, into the hemispheres for which $90^{\circ} \leq \theta \leq 180^{\circ}$ (henceforth to be called the "forward" hemisphere). Upon computing the power radiated into the "forward" and "rear" $\left(0^{\circ} \leq \theta \leq 90^{\circ}\right)$ hemispheres, by integrating the Poynting vector over the two hemispheres, we find that there is more power radiated into the forward hemisphere. This is equivalent to the statement that

$$
\int_{\pi / 2}^{\pi}\left|E_{r a d}\right|^{2} \sin \theta d \theta>\int_{0}^{\pi / 2}\left|E_{r a d}\right|^{2} \sin \theta d \theta .
$$

The effect of $\theta_{0}$ upon the appearance of the radiation pattern is marked only when the antenna has appreciable size relative to a wavelength. This has already been implied in the discussion of small spheres.

The asymmetry we have found when $\theta_{0} \neq 90^{\circ}$ is due, mathematically speaking, to the fact that when $\theta_{0} \neq 90^{\circ}$, both the even-numbered and odd-numbered modes $P_{n}^{1}(\cos \theta)$ are excited, whereas when $\theta_{0}=90^{\circ}$ only the odd-numbered modes are excited. The odd-numbered modes are symmetric about $\theta=90^{\circ}$, the even-numbered modes are antisymmetric, that is,

$$
\begin{gathered}
P_{n}^{1}\left(90^{\circ}+\delta\right)=-P_{n}^{1}\left(90^{\circ}-\delta\right), n \text { even } \\
P_{n}^{1}\left(90^{\circ}+\delta\right)=P_{n}^{1}\left(90^{\circ}+\delta\right), n \text { odd }
\end{gathered}
$$

\section{End Feed}

In figures 3, 4, and 5, discussed above, there are radiation patterns purporting to have the driving force at $\theta_{0}=0^{\circ}$. This calls for an explanation. Of course, as $\theta_{0}$ approaches 0 , the radiated power approaches zero for a finite applied voltage $V$. This is shown by our equations and agrees with the experience of experimental antenna workers who find that an antenna becomes a poor radiator as the feed point is moved toward the end. Some qualitative details on this point are given below. Although the radiation approaches zero, the radiation pattern approaches a stationary configuration. We are interested in obtaining this limiting pattern, which will serve as a good approximation for the patterns obtained when $\theta_{0}$ is not quite zero; that is, when the antenna is fed near one pole.

To accomplish this we must see what form eq 14 takes as $\theta_{0} \rightarrow 0$. The part containing $\theta_{0}$ is

$$
a\left(n, \theta_{0}\right) \equiv \frac{2 n+1}{2 n(n+1)} P_{n}^{1}\left(\cos \theta_{0}\right) \sin \theta_{0} .
$$

We wish to express $P_{n}^{1}\left(\cos \theta_{0}\right)$ as a Taylor's series of the form 


$$
P_{n}^{1}\left(\cos \theta_{0}\right)=g(n) \theta_{0}+h(n) \theta_{0}^{2}+\ldots .
$$

Write the well-known formula [10]

$P_{n}\left(\cos \theta_{0}\right)=\frac{1 \cdot 3 \cdot 5 \ldots(2 n-1)}{n !}\left[\left(\cos \theta_{0}\right)\right.$

$$
\begin{aligned}
& -\frac{n(n-1)\left(\cos \theta_{0}\right)^{n-2}}{(2 n-1) 2} \\
& \left.+\frac{n(n-1)(n-2)(n-3)\left(\cos \theta_{0}\right)^{n-4}}{(2 n-1)(2 n-3) 2 \cdot 4}+\cdots\right]
\end{aligned}
$$

and

$P_{n}^{1}\left(\cos \theta_{0}\right)=-\frac{d}{d \theta} P_{n}\left(\cos \theta_{0}\right)$

$$
\begin{aligned}
& =\frac{1 \cdot 3 \cdot 5 \ldots(2 n-1)}{n !} \sin \theta\left[n\left(\cos \theta_{0}\right)^{n-1}\right. \\
& -\frac{n(n-1)(n-2)\left(\cos \theta_{0}\right)^{n-3}}{(2 n-1) 2} \\
& \left.+\frac{n(n-1)(n-2)(n-3)(n-4)\left(\cos \theta_{0}\right)^{n-5}}{(2 n-1)(2 n-3) 2 \cdot 4}+\cdots\right]
\end{aligned}
$$

Then

$$
\begin{aligned}
g(n) & =\frac{1 \cdot 3 \cdot 5 \ldots(2 n-1)}{n !}\left[n-\frac{n(n-1)(n-2)}{(2 n-1) 2}\right. \\
& \left.+\frac{n(n-1)(n-2)(n-3)(n-4)}{(2 n-1)(2 n-3) 2 \cdot 4}+\cdots\right]
\end{aligned}
$$
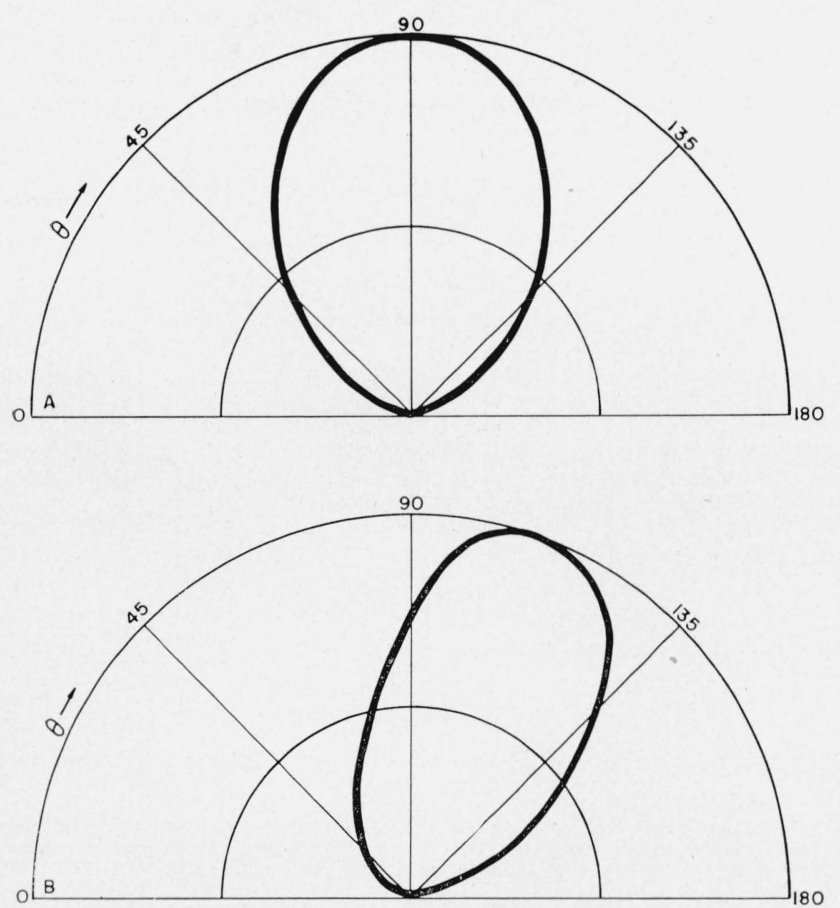

F1gure 3. Power radiation patterns for $k a=1$.

The patterns show $\left|E_{\text {rad }}\right|^{2}$ as a function of $\theta$. Both patterns are normalized to the same arbitrary maximum value of $\left|E_{\text {rad }}\right|^{2}$. A, $\theta_{0}=90^{\circ} ; \mathrm{B}, \theta_{0}=0^{\circ}$.
From this,

$$
\begin{aligned}
g(n+1)-g(n) & =(n+1)\left[\frac{1 \cdot 3 \cdot 5 \cdots(2 n-1)}{n !}\right. \\
& -\frac{1}{2} \frac{1 \cdot 3 \cdot 5 \ldots 2 n-3}{(n-2) !} \\
& \left.+\frac{1}{2 \cdot 4} \frac{1 \cdot 3 \cdot 5 \ldots(2 n-5)}{(n-4) !}+\cdots\right]
\end{aligned}
$$

But referring to eq 26, we see that the expression in square brackets in eq 28 is $P_{n}(1) \equiv 1$, so that

$$
g(n+1)-g(n)=n+1,
$$

$$
\text { or } \quad g(n)=\frac{n(n+1)}{2} \text {. }
$$
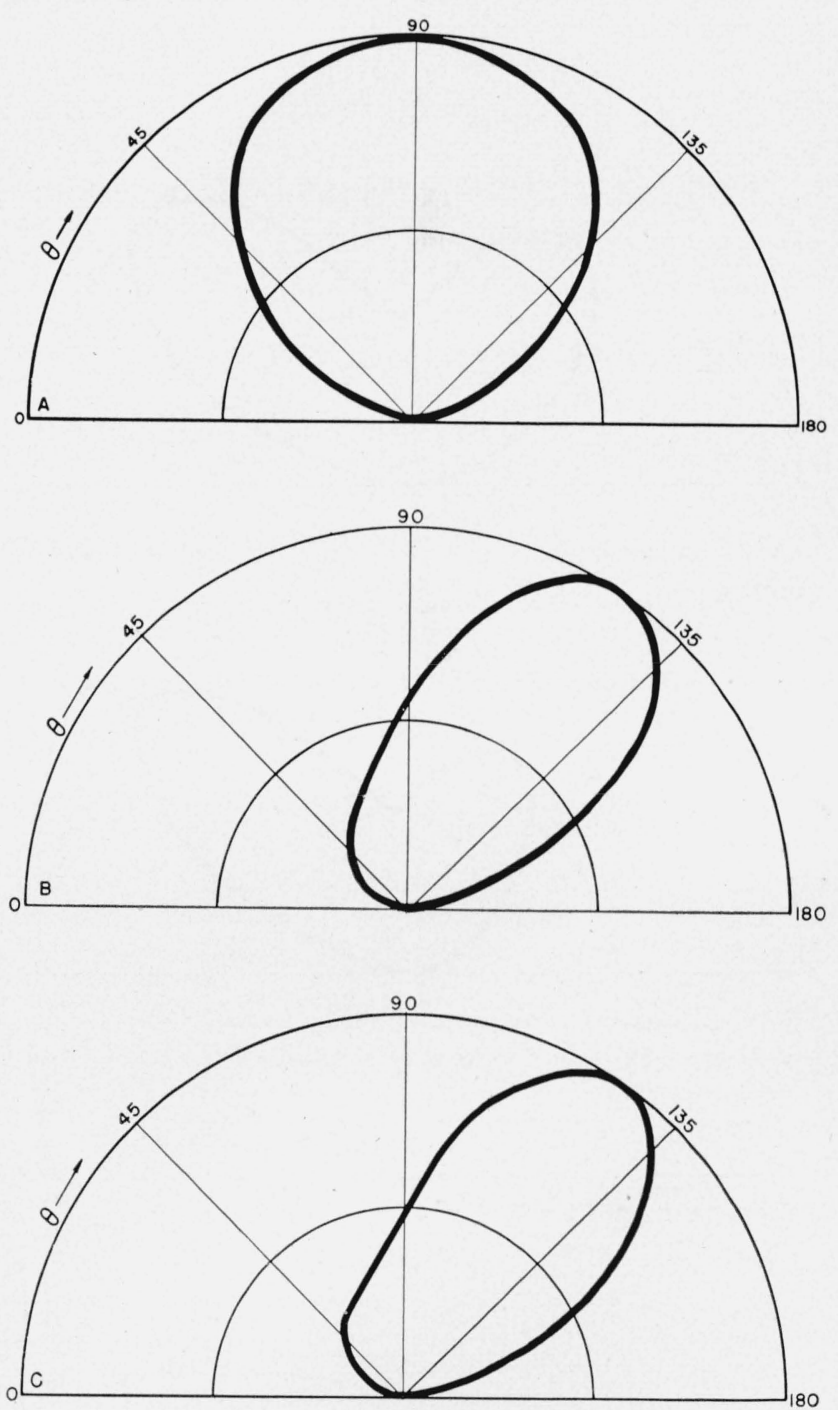

Figure 4. Power radiation patterns for $k a=1 \frac{1}{2}$.

A, $\theta_{0}=90^{\circ} ; \mathrm{B}, \theta_{0}=45^{\circ} ; \mathrm{C}, \theta_{0}=0^{\circ}$. The normalization is similar to that in figure 3 . 

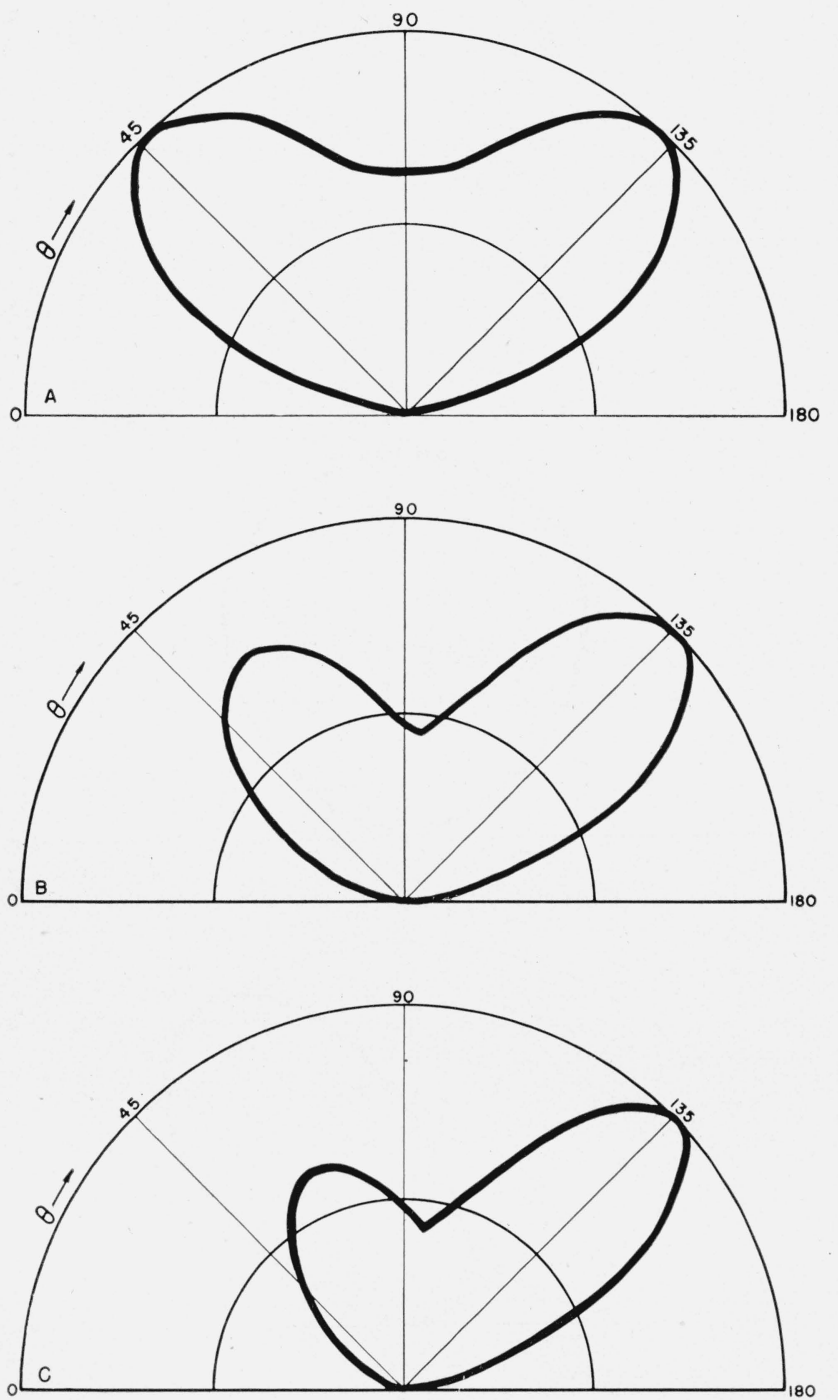

FIgURe 5. Power radiation patterns for $k a=2$. A, $\theta_{0}=90^{\circ} ; \mathrm{B}, \theta_{0}=45^{\circ} ; \mathrm{C}, \theta_{0}=0^{\circ}$. The normalization is similar to that in figure 3 .
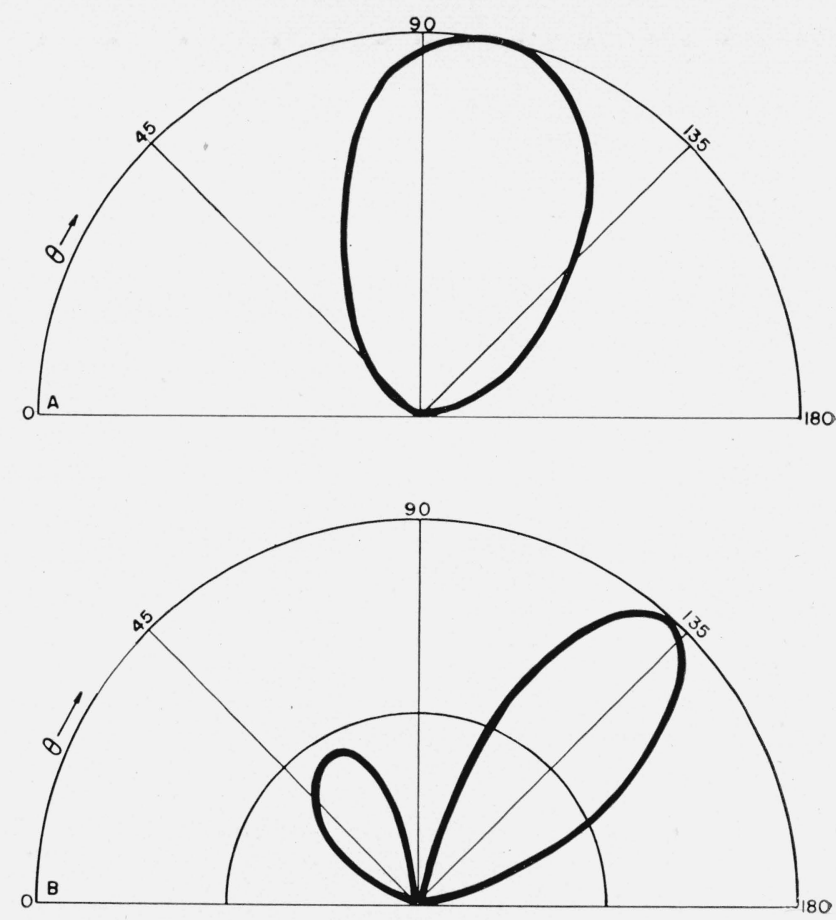

Figure 6. Power radiation patterns for end-fed cylinders.

Ratio of diameter to wavelength, 0.04 . A, Ratio of length to wavelength, $1 / 2 ; \mathrm{B}$, ratio of length to wavelenth, 1 . The normalization is similar to that in figure 3 .
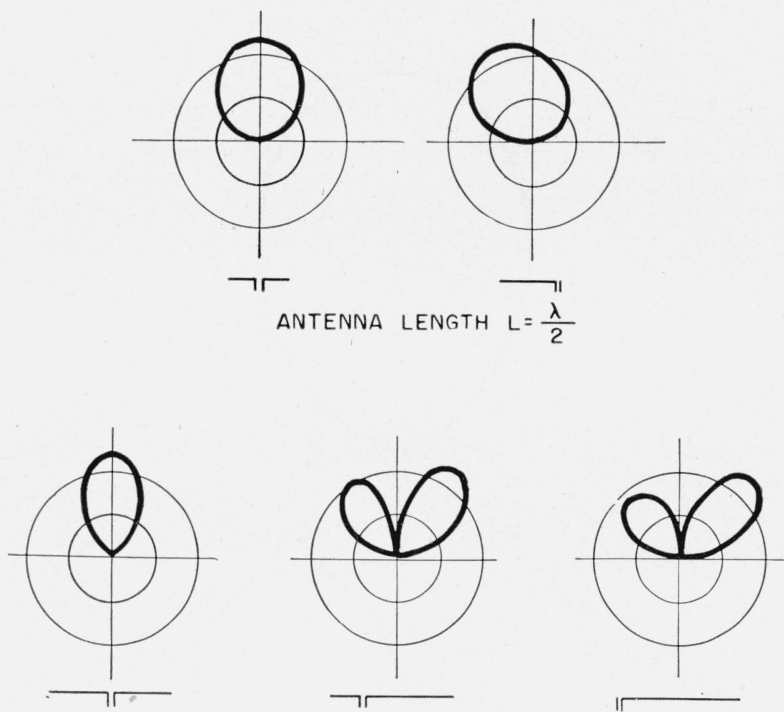

ANTENNA LENGTH L $=\lambda$

FIGURE 7. Effect of feed point upon radiation patterns of wire antennas.

Relative position of the feed point is shown under each pattern. Relative field strength $\left|E_{\text {rad }}\right|$ is shown. This material is adapted from figures 1 and 2 of reference [2] by permission of the McGraw-Hill Publishing Co. 


$$
\frac{\infty}{\infty} \frac{\infty}{\infty}
$$


Using (15), we then have, as $\theta_{0}$ approaches zero

$$
a\left(n, \theta_{0}\right)=\frac{(2 n+1)}{4} \theta_{0}^{2}+0\left(\theta_{0}\right)^{3} .
$$

Thus the limiting shape $S(\theta)$ of the radiation pattern as $\theta \rightarrow 0$ is given by

$$
S(\theta)=\sum_{1}^{\infty}(2 n+1) L(n, k a) P_{n}^{1}(\cos \theta) .
$$

Looking back at eq 19 and 20, we see that $S(\theta)$ converges. These patterns for $\theta_{0}=0$ are close approximations to the patterns obtained when the feed is near $\theta_{0}=0^{\circ}$. Among asymmetrically fed antennas, those having nearly end feed appear to be the most commonly used, so the results found for $\theta_{0} \approx 0$ are of especial interest.

\section{Related Experimental Results}

No experimental results for spherical antennas are known to the author. However, it is of considerable interest to note that the general type of behavior shown in figures 3,4 , and 5 , as to the effect of $\theta_{0}$ upon the pattern, has recently been found experimentally for wire antennas [2] and thick cylinders [1]. (Quantitatively, of course, we cannot compare such experiments with our theory). In those of the experiments in which the antenna lengths were of the order of a wavelength (so that the radiation patterns like those in figs. 3,4 , and 5 are relatively simple), the effect of moving $\theta_{0}$ toward $0^{\circ}$ was quite clearly reflected in the patterns; they have this characteristic lean with more than half the radiated power going into the forward hemisphere. For example, figure 6 shows radiation patterns found for two antennas consisting of cylindrical pipes for which the ratio of diameter $d$ to wavelength $\lambda$ was 0.04 , and whose ratios of length $L$ to $\lambda$ were $1 / 2$ and 1 , respectively. Each pipe contained a small gap near one end, across which the generator was connected. The general similarity of the patterns to those of figures $3 \mathrm{~b}$ and $5 \mathrm{c}$ is clear. Figure 7 shows some patterns obtained from figure 1 and figure 2 of reference [2], showing the same sort of effect for wires.

It is pleasing that our radiation pattern calculations for the asymmetrically fed spherical antenna show features that seem to be characteristic of a wide class of asymmetrically fed antennas and that have been experimentally observed.

\section{Large Spheres}

It is of interest to examine the radiation patterns for larger values of $k a$ (figs. 8, 9, 10). Here we find, as expected, increasingly complicated, multilobed, patterns. We find also that the "leaning" trend that exists for smaller antennas is no longer clear. One can no longer say that in the case of asymmetric feed the radiation pattern leans away from the feed point - the patterns have too many lobes, large ones appearing in both hemispheres. One might suspect, however, that even though the pattern trends are more complicated, that here to, as in the case of the smaller antennas, there is more power radiated into the forward hemisphere. However, even this is no longer true; in several cases an integration of Poynting's vector shows that less than half of the energy is radiated into the forward hemisphere even for $\theta_{0}=0$. For the larger antennas there does not seem to be any definite trend for the radiated energy to favor either hemisphere. Thus except for the smaller spheres, the location of the feed point is not reflected in any clear-cut way in power distribution in the hemispheres. These statements appear also to be true in the case of the longer asymmetrically fed wire antennas (figs. 3, 4, and 5 of reference [2]).

\section{Near-Polar Radiation}

The question arises: is there a simple characteristic of the radiation pattern that indicates unambiguously which side of the antenna contains the feed point? For the smaller antennas there is a characteristic lean which renders such identification possible, but this effect becomes unclear as the antenna increases in size. The hypothesis that there is always more power radiated into the forward hemisphere has also been shown above to be false. The present investigation has, however, revealed one simple feature that appears to be correlated unambiguously with the location of the feed point. 'This is a characteristic, to be discussed below, of the radiation in the near-polar directions, that is, the radiation in the directions near $\theta=0^{\circ}$ and $\theta=180^{\circ}$.

We have seen that $E_{r a d}$ is a superposition of the Legendre functions $P_{n}^{1}(\cos \theta)$. All these functions are of the form $b(n) \theta$, for sufficiently small $\theta$, where $b(n)$ is a constant depending upon $n$, so that $E_{r a d}$ is of the form $c_{1} \theta$ for small $\theta$. At the other pole $E_{\text {rad }}$ is of the form $c_{2} \psi$, where $\psi=\pi-\theta$. At $\theta=0$ and $\psi=0$, $E_{r a d}=0$. In general, $\left|c_{1}\right| \neq\left|c_{2}\right|$ except when the excitation is symmetrical $\left(\theta_{0}=90^{\circ}\right)$.

We have seen that for $\theta_{0}=0$ the shape of the pattern is given by eq 31 . Using eq 25 and 29 we find that for small $\theta$ this becomes

$$
S(\theta)=\sum_{n=1}^{\infty} \frac{(2 n+1) n(n+1)}{2} \theta L(n, k a)+0(\theta)^{2} .
$$

Thus we have

$$
\begin{aligned}
\frac{\left|c_{1}\right|^{2}}{Q^{2}}= & {\left[\sum_{n=1}^{\infty}(2 n+1) n(n+1) \operatorname{Re}: L(n, k a)\right]^{2}+} \\
& {\left[\sum_{n=1}^{\infty}(2 n+1) n(n+1) \operatorname{Im}: L(n, k a)\right]^{2}, }
\end{aligned}
$$

where $Q^{2}$ is a constant immaterial to the present discussion, and $R e$ and $I m$ indicate the real and imaginary part, respectively. Also, recalling the symmetry and anti-symmetry properties of $P_{n}^{1}(\cos \theta)$, 


$$
\begin{aligned}
\frac{\left|c_{2}\right|^{2}}{Q^{2}}= & {\left[\sum_{n=1}^{\infty}(-1)^{n+1}(2 n+1) n(n+1) \operatorname{Re}: L(n, k a)\right]^{2}+} \\
& {\left[\sum_{n=1}^{\infty}(-1)^{n+1}(2 n+1) n(n+1) \operatorname{Im}: L(n, k a)\right]^{2} }
\end{aligned}
$$

These definitions of $\left|c_{1}\right|$ and $\left|c_{2}\right|$ are for $\theta_{0}=0$; for other values of $\theta_{0}$ there are corresponding definitions.

The hypothesis here presented, with considerable evidence, is that if $\theta_{0}<90^{\circ},\left|c_{2}\right|>\left|c_{1}\right|$. This has been found to be true in all the spherical antenna patterns that have been computed. This can be seen, for example, in figures 3, 4, 5, 8, 9, and 10. (It has also been found in the experimental work on thick cylindrical antennas, and is also apparently true in the case of the 16 radiation patterns given in reference [2] for wire antennas.)

For the interesting case $\theta_{0}=0^{\circ}$, we have calculated $\left|c_{1}\right|$ and $\left|c_{2}\right|$ from (32) and (33) for a considerable number of values of $k a$. The results are plotted in figure 11 as a function of $k a$. In this figure the points that have been computed have been clearly indicated. The dashed curve shown is suggested by the points. We see that $\left|c_{2}\right| /\left|c_{1}\right|$ has the value 1 at $k a=0$, and appears to have a generally increasing trend. Oscillations are superposed upon this trend, but there is no evidence that the curve dips down as far as the value 1 except at $k a=0$. Thus, particularly in the case of end feed, all the evidence so far seems to support our hypothesis that the smaller of the two quantities $\left|c_{2}\right|$ and $\left|c_{1}\right|$ indicates unambiguously which side of the antenna contains the feed point. However, on account of the complicated analytic form of $\left|c_{1}\right|$ and $\left|c_{2}\right|$, illustrated in eq 32 and 33, a complete proof of this statement has not yet been found.

\section{Crude Model of an End-Fed Antenna}

It is perhaps of some interest to mention that we have been able in the course of this investigation to prove that $\left|c_{2}\right| /\left|c_{1}\right|>1$ in the case of a very crude model of an end-fed antenna. Consider the radiation field of a linear current distribution

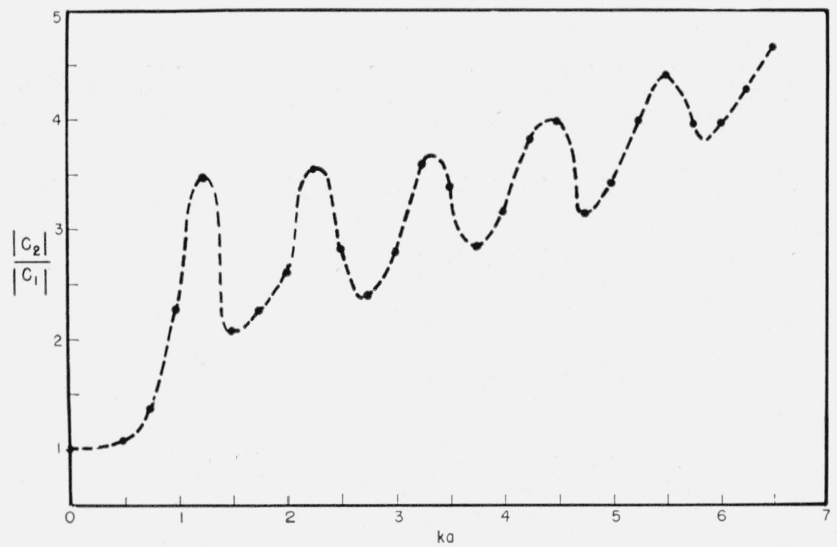

Figure 11. $\left|c_{2}\right| /\left|c_{1}\right|$ as a function of ka for $\theta_{0}=0^{\circ}$. Computed points are shown as dots.

$$
I=I_{0} e^{j k z}+R I_{0} e^{-j k z},
$$

whose length is $L$, and which is centered at the origin $z=0$. This distribution consists of a wave travelling in the negative $z$ direction and a return wave that represents the original wave subjected at $-\mathrm{L} / 2$ to a complex reflection coefficient $R$. We shall restrict ourselves to the condition $|R|<1$. The time factor $e^{j \omega t}$ has been suppressed. Such a distribution may be expected on the basis of rough physical reasoning to give radiation patterns similar to those found for actual end-fed antennas. This can be seen in more detail by considering the well-known radiation pattern [11] obtained from the first term of (34), a pure travelling wave. A typical pattern is shown in figure 12 . It will be seen that the main radiation is at an acute angle with the direction of the current flow. The distribution (34) may be said to give rise to two such radiation patterns of unequal magnitude, pointed in essentially opposite directions, and superposed (the complex values of $E_{\text {rad }}$ are added). The resultant pattern may be expected to be very much like the actual patterns for end-fed antennas. In the case of the cylinders, for example, it was found some time ago [1] that the patterns can be quite closely duplicated by proper choice of $R$.

We shall now prove that for this simple model $\left|c_{2}\right| /\left|c_{1}\right|>1$. It is convenient here to derive $\boldsymbol{E}$ from the vector potential $\boldsymbol{A}$, according to the standard equations. Only a $z$ component of $\boldsymbol{A}$ is present.

We have, since $\partial / \partial t=j \omega$

$$
\boldsymbol{E}=-j \omega \mu \boldsymbol{A}+\frac{1}{j \omega \epsilon} \nabla(\nabla \cdot \boldsymbol{A})
$$

also

$$
\boldsymbol{A}=A_{z}=\frac{1}{4 \pi} \int_{-L / 2}^{L / 2} \frac{I(z) e^{-j k r}}{r} d z,
$$

where $r$ is the distance between the point $P$ at which we are computing the field and the point $z$. If $P$ is a remote point, $r \approx r_{0}-z \cos \theta$, where $r_{0}$ is the distance from $P$ to the origin.

We may replace $r$ in the denominator of the integrand by $r_{0}$ but must keep the more accurate expression in the exponential factor. We have then

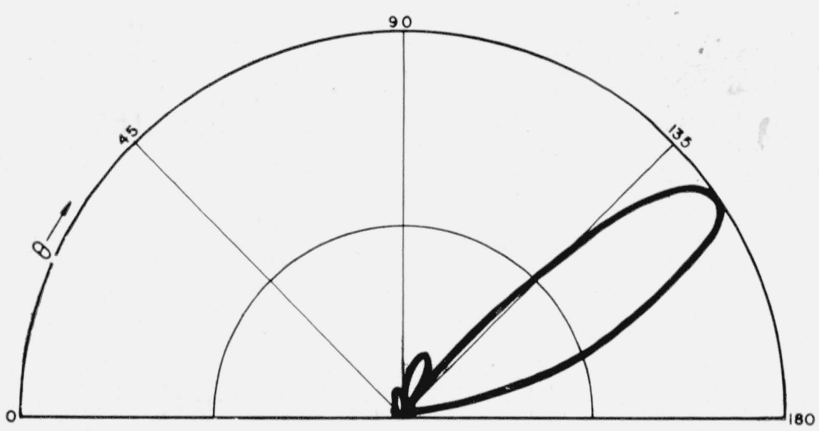

Figure 12. Power radiation pattern of travelling wave of current.

Length $L_{-}$of current distribution, $2 \lambda$. The wave is travelling toward the right. 


$$
\begin{aligned}
A_{2} & =\frac{I_{0} e^{-j k r_{0}}}{4 \pi r_{0}} \int_{-L / 2}^{L / 2}\left[e^{j k z}+R e^{-j k z}\right] e^{j k z \cos \theta} d z, \\
& =\frac{I_{0} e^{-j k r_{0}}}{4 \pi r_{0}}\left\{\frac{e^{j k(L / 2)(1+\cos \theta)}-e^{-j k(L / 2)(1+\cos \theta)}}{j k(1+\cos \theta)}\right.
\end{aligned}
$$

also

$$
\begin{gathered}
\left.+\frac{R\left[e^{j k(L / 2)(\cos \theta-1)}-e^{-j k(L / 2)(\cos \theta-1)}\right]}{j k(\cos \theta-1)}\right\} \\
=\frac{I_{0} e^{-j k r} 0}{2 \pi r_{0} k}\left\{\frac{\sin (k L / 2)(\cos \theta-1)}{\cos \theta+1}\right. \\
\left.+\frac{R \sin (k L / 2)(\cos \theta-1)}{\cos \theta-1}\right\} .
\end{gathered}
$$

The radiation field, it turns out, is found from the first term of (35):

$$
E_{r a d}=-j \omega \mu A_{\theta}=j \omega \mu A_{z} \sin \theta
$$

so

$$
\begin{aligned}
E_{\text {rad }}= & \left.\frac{-\omega \mu I_{0} e^{-j k r_{0}} \sin \theta}{2 \pi r_{0} j k}\right\} \frac{\sin \frac{k L}{2}(\cos \theta+1)}{\cos \theta+1}+ \\
& \left.\frac{R \sin \frac{k L}{2}(\cos \theta-1)}{\cos \theta-1}\right\} .
\end{aligned}
$$

The essential part of this is

$$
\sin \theta\left\{\frac{\sin A(\cos \theta+1)}{\cos \theta+1}+\frac{R \sin A(\cos \theta-1)}{\cos \theta-1}\right\}
$$

where $A \equiv k L / 2$.

Now as $\theta \rightarrow 0$, the expression in brackets becomes

$$
\frac{\sin 2 A}{2}+A R \equiv \frac{c_{1}}{Q_{1}}
$$

and as $\theta \rightarrow \pi$, we have

$$
A+R \frac{\sin 2 A}{2} \equiv \frac{c_{2}}{Q_{1}}
$$

where, as we see, $c_{1}$ and $c_{2}$ are defined as for the spherical antenna. $Q_{1}$ is a constant.

We shall now show that $\left|c_{2}\right|>\left|c_{1}\right|$. Since $R$ is taken to be a complex number we write $R \equiv|R| e^{j \alpha}$. Then

$$
\begin{aligned}
& \frac{\left|c_{2}\right|^{2}}{\left|Q_{1}\right|^{2}}=A^{2}+\frac{|R|^{2} \sin ^{2} 2 A}{4}+A|R| \cos \alpha \sin 2 A \\
& \frac{\left|c_{1}\right|^{2}}{\left|Q_{1}\right|^{2}}=A^{2}|R|^{2}+\frac{\sin ^{2} 2 A}{4}+A|R| \cos \alpha \sin 2 A \\
& \frac{\left|c_{2}\right|^{2}-\left|c_{1}\right|^{2}}{\left|Q_{1}\right|^{2}}=\left(A^{2}-\frac{\sin ^{2} 2 A}{4}\right)\left(1-|R|^{2}\right),
\end{aligned}
$$

which is never negative and is zero only when $A=\mathrm{O}$. Thus for this simple model $\left|c_{2}\right|>\left|c_{1}\right|$. It is also interesting to note that the general behavior of the quantity $\left|c_{2}\right| /\left|c_{1}\right|$ for this model is rather similar to that in the case of the spherical end-fed antenna. Consider the ratio

$$
\delta^{2} \equiv \frac{\left|c_{2}\right|^{2}}{\left|c_{1}\right|^{2}}=\frac{A^{2}+\left(|R|^{2} / 4\right) \sin ^{2} 2 A+A|R| \cos \alpha \sin 2 A}{A^{2}|R|^{2}+\frac{\sin ^{2} 2 A}{4}+A|R| \cos \alpha \sin 2 A},
$$

Examination of this expression shows that $\delta$ has the value 1 at $A=0$, begins to increase slowly with increasing $A$, then increases rapidly and begins to oscillate, always remaining greater than 1 . If $|R|$ is a constant independent of $A, \delta$ approaches in an oscillating manner the asymptotic value $1 /|R|$. If, however, $|R|$ decreases as $A$ increases, $\delta$ has a continually increasing trend with superimposed oscillations. This is the type of behavior shown by $\left|c_{2}\right| /\left|c_{1}\right|$ for the sphere (and presumably for other types of end-fed antennas). It may be noted that if one wished to simulate an end-fed antenna by this crude model, it would be in keeping with general ideas on antenna radiation to have $|R|$ decrease with increasing length of the antenna. A better model would probably have the current wave attenuated gradually along the antenna, rather than suddenly at the end.

\section{Current and Admittance}

Having discussed the radiation field in some detail, we now consider questions of admittance and current. The admittance presented to the applied voltage is determined by the value of the current at the feed point. Since we have postulated a perfectly conducting antenna, there will be at the surface a discontinuity in $H_{\phi}$ and a corresponding surface current density $J_{\theta}$, related to $H_{\phi}$ at any point on the surface by

$$
J_{\theta}=-\left.H_{\phi}\right|_{r=a}
$$

The direction of current flow is along meridian lines. The total current $I_{\theta}$ at colatitude $\theta$ is

$$
I_{\theta}=2 \pi a \sin \theta J_{\theta}=-\left.2 \pi a \sin H_{\phi}\right|_{r=a}
$$

Using (4a) and (9), we find

$$
\begin{aligned}
I_{\theta}= & 2 \pi a \sin \theta j \omega \epsilon V \sum_{n=1}^{\infty} a_{n} \\
& \frac{H_{n+1 / 2}^{(2)}(k a)}{n H_{n+(1 / 2)}^{(2)}(k a)-k a H_{n+(1 / 2)}^{(2)}(k a)} P_{n}^{1}(\cos \theta) \\
= & \sum_{n=1}^{\infty} I_{\theta}(n)
\end{aligned}
$$


where $I_{\theta}(n)$ is the partial current associated with the $n$th mode. Rearranging (43) a bit, this takes the form

$$
\begin{aligned}
I_{\theta}(n)= & 2 \pi a \sin \theta J_{\theta}(n)= \\
& \frac{V \pi}{Z_{0}} a\left(n, \theta_{0}\right) K(n, k a) P_{n}^{1}(\cos \theta) \sin \theta,
\end{aligned}
$$

where

$$
\frac{1}{Z_{0}} \equiv \frac{\omega \epsilon}{k} \equiv \sqrt{\frac{\epsilon}{\mu}}
$$

and

$$
K(n, k a) \equiv \frac{j}{\frac{n}{k a}-\frac{H_{n-1 / 2}^{(2)}(k a)}{H_{n+1 / 2}^{(2)}(k a)}} .
$$

The relation between each partial current and the corresponding partial radiation field $E_{\text {rad }}(n)$ is

$$
J_{\theta}(n)=\frac{I_{\theta}(n)}{2 \pi a \sin \theta}=\frac{-j^{n+1} k r \sqrt{\frac{\pi}{2 k a}}}{2 Z_{0} e^{-j k r}} H_{n+1 / 2}^{(2)}(k a) E_{\text {rad }}(n),
$$

a relation that is independent of the excitation function $a\left(n, \theta_{0}\right)$. This relation means that the $n$th current mode is associated with or "causes" a corresponding $n$th radiation mode, independently of any other modes which, as a result of a particular form of excitation, may be present.

Defining the admittance $Y$ as

we find

$$
Y \equiv \sum_{n=1}^{\infty} Y(n) \equiv \frac{I_{\theta_{0}}}{V}
$$

$$
\begin{aligned}
Y= & \sum_{n=1}^{\infty} Y(n)=\sum_{n=1}^{\infty} \frac{I_{\theta_{0}}(n)}{V}= \\
& \sum_{n=1}^{\infty} \frac{\pi}{Z_{0}} a\left(n, \theta_{0}\right) K(n, k a) P_{n}^{1}\left(\cos \theta_{0}\right) \sin \theta_{0} .
\end{aligned}
$$

We see that the applied voltage "sees" an infinite number of partial admittances in parallel.

The real and imaginary parts of the function $K(n, k a)$, which represents the effect of the size of the sphere in the expressions for current and admittance are plotted in figure 13 for the first few modes. We note that for a given value of $k a$, values of $n$ appreciably greater than $k a$ all contribute only negligibly to $R e: K(n, k a)$, whereas in the case of $I m: K(n, k a)$ the contributions of the higher modes fall off only slowly with increasing $n$. This is related to the fact that, as we have seen, the radiation field is determined by only a relatively small number of modes; the part of $Y$ that accounts for the radiation is the real part of conductance $C$. On the other hand, the part of the field that is reactive, rather than radiative, is associated with the imaginary part of $Y$, the susceptance $S$.
Upon examining the series (49) for current and admittance, it is found that this series is divergent. [Therefore for $\theta=\theta_{0}$ (44) is divergent]. Using for $a\left(n, \theta_{0}\right)$ the expression (15) referring to the deltafunction excitation, we have

$$
Y=\sum_{n=1}^{\infty} \frac{\pi}{Z_{0}} \frac{2 n+1}{2 n(n+1)}\left[P_{n}^{1}\left(\cos \theta_{0}\right)\right] \sin ^{2} \theta_{0} \frac{j}{\frac{n}{k a}-\frac{H_{n-1 / 2}^{(2)}(k a)}{H_{n+1 / 2}^{(2)}(k a)}} .
$$

Using the expressions (20) and (18), one finds that for sufficiently high $n$ the general term of the series approaches

$$
\frac{j(2 n+1) k a}{Z_{0} n(n+1)} \cos ^{2}\left[\left(n+\frac{1}{2}\right) \theta_{0}+\frac{\pi}{4}\right] \sin ^{2} \theta_{0} .
$$

Consider the series whose $n$th term is the expression (51). We shall show that for the infinite set of values of $\theta_{0}$ given by

$$
\theta_{0}=2 \pi \frac{p}{q},(p, q \text { integer, } q>p),
$$

the series diverges. For our purposes, this is a sufficient indication of divergence over the range of $\theta_{0}$.

The $\cos ^{2}$ factor, which is nonnegative, will repeat itself when $n$ increases by $q$. Now choose $n=r$ such that

$$
\cos ^{2}\left[\left(r+\frac{1}{2}\right) \theta_{0}+\frac{n}{4}\right]=c>0 .
$$

Then, considering only the values of $n$ given by

$$
r, r+q, r+2 q, \text { etc., }
$$

we examine the series

$$
c\left\{\frac{2 r+1}{r(r+1)}+\frac{2(r+q)+1}{(r+q)(r+q+1)}+\frac{2(r+2 q)+1}{(r+2 q)(r+2 q+1}+\cdots\right\}
$$

Dividing the $n$th term $u_{n}$ of the series by $u_{n+1}$ (Raabe's test [12]), we find

$$
\frac{u_{n}}{u_{n}+1}=1+\frac{1}{n}+0\left(\frac{1}{n^{2}}\right)
$$

showing that (52) is divergent. Therefore the series whose general term is (51) also diverges. The higher order terms in (20) and (18), which have been neglected in writing down (51), do not alter this conclusion. Thus the series for the admittance $Y$ does not converge. It is to be noted, however, that it is the imaginary part of $Y$, the susceptance $S$, that diverges. The real part, the conductance $C$, converges rapidly, as implied above.

The nonconvergence of $S$ is due to the deltafunction excitation that has been assumed. The 
susceptance becomes that of an infinite capacity, which is understandable; concentrating the applied force $E^{\prime}$ along a line at $\theta=\theta_{0}$ is equivalent to maintaining a potential difference across an infinitesimal gap at $\theta=\theta_{0}$. The infinitesimal gap has infinite capacity. (This gap terminology, represents, as J. C. Slater [13] has pointed out, another way of interpreting physically the type of boundary condition we are using, [explained in Part II]. In this approach, one considers that the driving field is applied tangentially across a small gap at the surface of the sphere and that solutions of the wave equation are being sought such that the tangential electric field will reduce to the applied field across the gap and to zero everywhere on the metallic surface.)

When the field $E^{\prime}$ is considered to be applied across a finite region, the high order terms in $a\left(n, \theta_{0}\right)$ decrease much more rapidly than in (11), which is for the infinitesimal case. For example if $E^{\prime}$ is constant $=V / a \Delta \theta$ over a small finite zone $\Delta \theta$ centered at $\theta_{0}$, then our general expression (7) for $a\left(n, \theta_{0}\right)$ reduces to

$$
\begin{aligned}
a\left(n, \theta_{0}\right) & =\frac{2 n+1}{2 n(n+1) \Delta \theta} \int_{\theta_{0}-(\Delta \theta / 2)}^{\theta_{u}+(\Delta \theta / 2)} P_{n}^{1}(\cos \theta) \sin \theta d \theta \\
& =-\frac{2 n+1}{2 n(n+1) \Delta \theta} \int_{\theta_{0}-(\theta \Delta / 2)}^{\theta_{0}+(\Delta \theta / 2)} \frac{d}{d \theta} P_{n}(\cos \theta) \sin \theta d \theta
\end{aligned}
$$

For small $\Delta \theta$, it will be sufficiently accurate in (54) to take $\sin \theta$ outside of the integral sign and give it
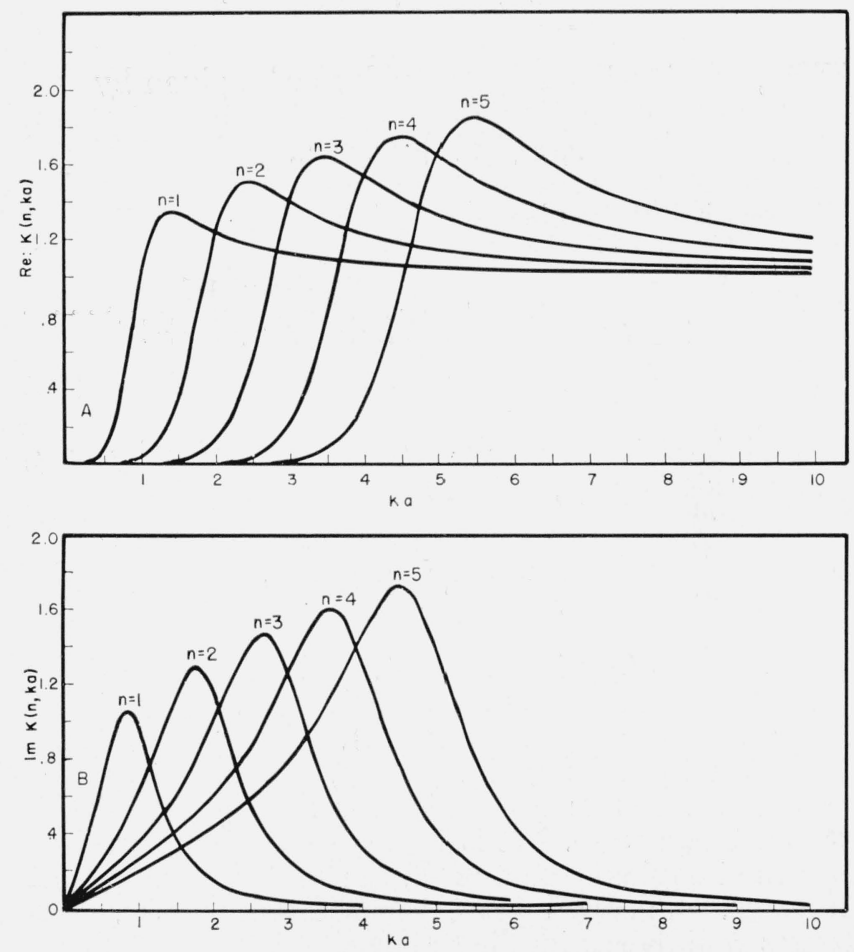

FiguRE $13 . \quad K(n, k a)$ as a function of $k a$ for several values of $n$. $\mathrm{A}$, real part; B, imaginary part. the value $\sin \theta_{0}$ (or some other average value). We then have

$$
\begin{aligned}
a\left(n, \theta_{0}\right) \approx & \frac{(2 n+1) \sin \theta_{0}}{2 n(n+1) \Delta \theta}\left[P_{n} \cos \left(\theta_{0}-\frac{\Delta \theta}{2}\right)-\right. \\
& \left.P_{n} \cos \left(\theta_{0}+\frac{\Delta \theta}{2}\right)\right] .
\end{aligned}
$$

For the first few values of $n$ this reduces very closely to $(11)$.

For high values of $n$, where the functions $P_{n}^{1}(\cos \theta)$ vary appreciably over the zone $\Delta \theta$, (55) begins to differ from (11). The asymptotic behavior of (55) for large $n$ now makes the series (49) converge. We find [8] that the $n$ dependence of $P_{n}(\cos \theta)$ for the large $n$ is $n^{-1 / 2}$ instead of $n^{1 / 2}$ for $P_{n}^{1}(\cos \theta)$ as in (20). It is easily seen that this change will make the $Y$ series converge.

This discussion indicates that the theoretical results for radiation field and conductance obtained with the delta-function excitation will not differ appreciably from the results for a finite $\Delta \theta$. This important conclusion is paralleled in the experimental field, where it is rather well known that the radiation pattern and radiation conductance of an antenna are

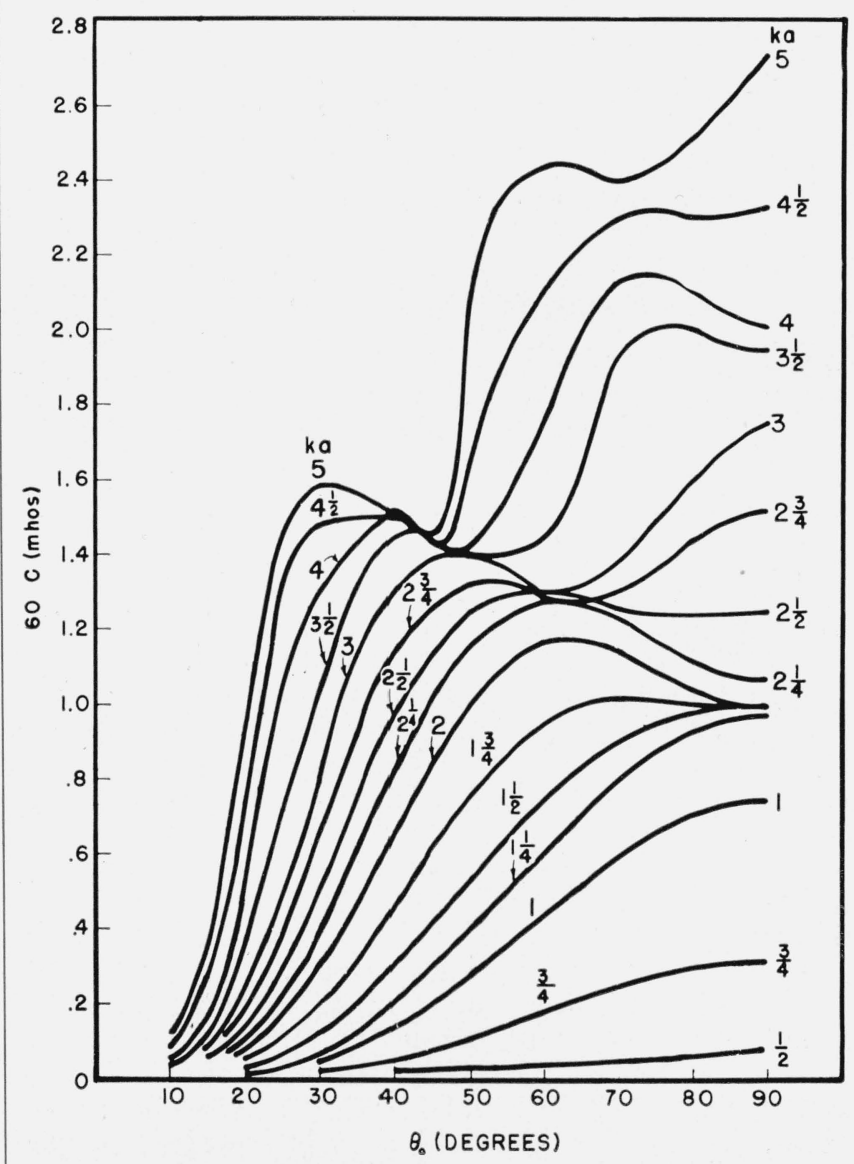

Figure 14. Radiation conductance, $C$, as a function of $\theta_{0}$, for several values of ka. 
very little affected by the size of the gap. The present work indicates in a sense the "reason" for this. It also tends to show that the idealized delta-function approach is capable of finding answers for radiation patterns and conductance that will agree with those found experimentally. On the other hand, the divergence of the imaginary part of the admittance indicates that these calculations do not throw too much light on the values of the susceptance that may be expected in practice. The values of this quantity, as is also well known experimentally, depend very much upon the details of the feed. Antenna calculations such as those in the present report severely schematize the feed conditions, so they cannot be expected to yield much information on susceptance.

As discussed above, we cannot plot the susceptance but confine ourselves to drawing graphs of the conductance, $C$, (fig. 14). We could, of course, attempt to plot the sum of the first $m$ partial susceptances, where $m$ is a number such that the contributions above $m$ would not be appreciable when $\Delta \theta$ is finite, and so that the contributions below $m$ would not be altered appreciably when $\Delta \theta$ is finite. This, however, is difficult because the high-order terms fall off extremely slowly. There are some qualitative conclusions about susceptance we can draw from the equations $(47,49,50$, and 55), and graphs (fig. 13). For $k a$ considerably less than 1 , the admittance will be primarily a capacitative susceptance, the conductance being small compared to the susceptance. For infinitesimal $\Delta \theta$ we see that the susceptance for a sphere of any size is always capacitative. For small but finite $\Delta \theta$, this will in general still be true, except possibly for $k a$ very large.

The general trend of $C$, as disclosed in figure 14, is to start with the value zero at $k a=0$, rise rapidly, and then go through $0,1,2$, or more secondary maxima (the number depending upon $k a$ ) before reaching its value at $\theta_{0}=90^{\circ}$. The trend near $\theta_{0}=0$ is of interest. From eq 50 we find that this trend is as $\theta^{4}{ }_{0}$. This is perhaps the first time in the literature that such a problem has received a

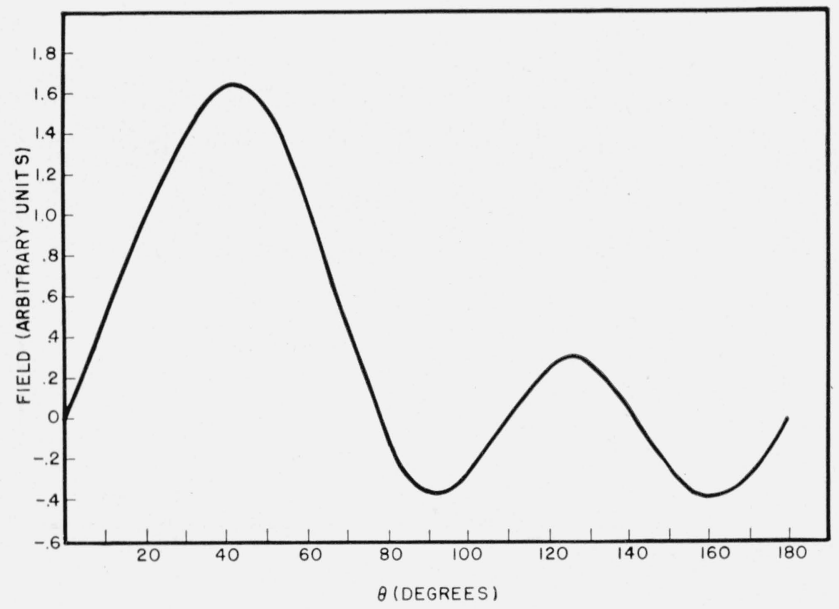

Figure 15. Sum of lowest five modes of applied field, for $k a=2$ and $\theta_{0}=45^{\circ}$. clear-cut answer. The corresponding question for wire antennas has, it is believed, not yet been solved.

For relatively small spheres, $k a<1$, the trend of $C$ is very closely as $\sin ^{4} \theta_{0}$ for all positions of the feed point. The reason for this is that the first mode that gives $\sin ^{4} \theta_{0}$ exactly, accounts for most of the conductance (and radiation).

The secondary maxima found for the larger antennas are of interest. Since for a given applied voltage $V e^{j \omega t}$ the time-average power radiated is $1 / 2 V^{2} C$, that is, proportional to $C$, we find in some cases that one gets more radiated power for a given voltage by feeding the antenna off center than for center feed. The local stationary regions on the $C$-vs- $\theta_{0}$ curve indicate locations on the antenna where the value of $C$ is not sensitive to the location of the feed point.

In general, we see that keeping $\theta_{0}$ constant while increasing $k a$, increases $C$. There are several quite stationary spots on the $C$-vs- $k a$ curve for given $\theta_{0}$. For $\theta_{0}=90^{\circ}, C$ is very flat from $k a=11 / 4$ to $k a=2$. For $\theta_{0}=60^{\circ}$, we find such a flat region for $k a=2$ to 3 , and for $\theta_{0}=45^{\circ}, C$ is virtually constant for $k a=3$ to 5 .

Some or all of the above features are probably to be found, qualitatively, even for nonspherical asymmetrically fed antennas. Experimentally (and theoretically), however, this seems to be a virtually unexplored field.

It may be noted that the first few modes into which the applied field is analyzed, which, as we have seen are primarily responsible for the form of the radiation pattern and the amount of radiated power, represent, when summed, a field that is very far from being localized at the "feed point". That is, we may say that this distributed applied field produces essentially the same radiation as the concentrated applied field. An example of this is shown in figure 15 , which shows the sum of the first five modes of the applied field for $k a=2$ and $\theta_{0}=45^{\circ}$. These modes account for practically all the radiation and determine the shape of the radiation pattern. The higher modes are associated with reactive components of the field that are localized near the antenna and whose importance is in meeting the boundary conditions at the antenna, including feed conditions. Similarly the current on the antenna that is associated with the first few modes and that may be considered "responsible" for the radiation, is very much different from the total current, which includes the higher reactive modes used in satisfying the local conditions.

\section{Conclusions}

An idealized treatment of the asymmetrically fed spherical antenna has been given that produces detailed results for the radiation properties as a function of size of antenna and location of the feed point. Reasons are given for believing that the idealization of the feed conditions does not prevent the theory from giving experimentally pertinent answers for the radiation properties. Some of the features show 
qualitative agreement with experimental work on nonspherical antennas. The theory should be useful as a rough guide for experiments with other types of asymmetrically fed antennas, particularly those whose ratio of diameter to length is fairly large.

The author thanks K. F. Herzfeld, Head of the Physics Department of Catholic University of America, for his generous help and guidance throughout the course of this work. Gratitude is also expressed to C. H. Page, of this Bureau, for many stimulating and enlightening discussions and for much kind encouragement and help. The entire paper has been read with critical care and valuable suggestions by the above named, by J. Mendousse and D. Beard, of Catholic University, and by J. J. Freeman, of this Bureau. To all these deep appreciation is expressed. Thanks are also extended to A. V. Astin and W. S. Hinman, Jr., of this Bureau, for general encouragement and cooperation. The generous secretarial and other assistance of Marion B. Teele is gratefully acknowledged.

\section{References}

[1] C. H. Page, R. D. Huntoon, and P. R. Karr, Radiation patterns of thick end-fed antennas; a talk presented at meetings of the Institute of Radio Engineers at Washington, D. C., May 1946, and New York, Feb. 1947.

[2] D. C. Cleckner, Effect of feed on patterns of wire antennas, Electronics 20, 103 (1947)

[3] F. E. Terman, Radio engineer's handbook, first ed., chapter 11 (McGraw-Hill Book Co., New York, N. Y., 1943).

[4] W. S. Hinman and Cledo Brunetti, Radio proximity fuze design, J. Research NBS 37, 1 (1946) RP1723; C. H. Page and A. V. Astin, Survey of proximity fuze development, Am. J. Phys. 15, No. 2, 95 (1947).

[5] J. A. Stratton and L. J. Chu, Forced oscillations of a conducting sphere, J. Applied Phys. 13, 236 (1941).

[6] E. T. Whittaker and G. N. Watson, A course of modern analysis, 4th ed., p. 324 (Cambridge Univ. Press, 1940).

[7] A. Sommerfeld, Partial differential equations of physics, p. 119 (Academic Press, New York, N. Y., 1949).

[8] E. W. Hobson, Spherical and ellipsoidal harmonics, p. 303 (Cambridge Univ. Press, 1931).

[9] J. A. Stratton, Electromagnetic theory, first ed., p. 436 (McGraw-Hill Book Co., New York, N. Y., 1941).

[10] T. M. MacRobert, Spherical harmonics, p. 86 (E. P. Dutton and Co., New York, N. Y., 1927).

[11] J. Aharoni, Antennae, p. 225 (Oxford Univ. Press, 1946).

[12] T. M. MacRobert, Functions of a complex variable, p. 77 (MacMillan and Co. Ltd., London, 1938).

[13] J. C. Slater, Microwave transmission, first ed., p. 221 (McGraw-Hill Book Co., New York, N. Y., 1942).

Washington, February 9, 1951. 